\title{
Chapter 5 \\ Transfer to Wheat of Potentially New Stem Rust Resistance Genes from Aegilops speltoides
}

\author{
Ian Dundas, Dawn Verlin, Peng Zhang, Yue Jin, Jacob Manisterski, \\ and Rafiqul Islam
}

\begin{abstract}
Stem rust resistance genes have been found in four different sources of Aegilops speltoides. These include diploid accessions AEG357-4 and AEG874-60 and the amphiploids Chinese Spring/Ae. speltoides TA8026 and TS01. Stem rust resistance was mapped to the $2 \mathrm{~S}$ chromosomes derived from each of these lines. The previously reported 2B-2S\#3 translocation derived from AEG357-4 was found to carry two stem rust resistance genes, here temporarily named SrAes $2 t$ and SrAes $3 t$. The resistance genes found on the $2 \mathrm{~S}$ chromosomes each derived from TA8026, TS01 and AEG874-60 are named SrAes4t, SrAes5t and SrAes6t, respectively. Lines carrying genes $\operatorname{SrAes} 2 t$ and $\operatorname{SrAes} 3 t$ are being distributed to wheat breeding programs around the world.
\end{abstract}

Keywords Aegilops speltoides $\bullet$ Resistance genes $\bullet$ Stem rust

\section{Introduction}

The fungal disease stem rust, caused by Puccinia graminis f. sp. tritici, has long been a scourge of wheat crops since the beginnings of agriculture. Uredia have even been found on ancient wheat spikelets estimated at 3,300 years old (Kislev 1982).

\footnotetext{
I. Dundas $(\bowtie) \bullet$ D. Verlin $\bullet$ R. Islam

School of Agriculture, Food and Wine, University of Adelaide, Waite Rd, Urrbrae,

SA 5064, Australia

e-mail: ian.dundas@adelaide.edu.au

P. Zhang

Plant Breeding Institute, The University of Sydney, 107 Cobbitty Rd, Cobbitty,

NSW 2570, Australia

Y. Jin

USDA-ARS Cereal Disease Laboratory, University of Minnesota, St Paul, MN 55108, USA

J. Manisterski

Institute for Cereal Crops Improvement, Tel Aviv University, Ramat Aviv 69978, Israel
} 
Recently, the appearance and spread of mutants of the Ug99 stem rust lineage (summarized in Mago et al. 2013) has raised the awareness of the need to search for new resistance genes for use in wheat cultivars. Most of the currently named stem rust genes in wheat originate from related species (e.g., Secale cereale, Triticum monococcum, T. turgidum, T. timopheevii, Thinopyrum ponticum, Aegilops speltoides, Ae. tauschii and Ae. ventricosum). Many of these genes (e.g., Sr2, Sr22, Sr24, Sr26, Sr36 and Sr38) are widely used in agriculture.

The diploid species Ae. speltoides Tausch. $(2 \mathrm{n}=2 \mathrm{x}=14)$ has provided several resistance genes against stem rust, namely Sr32, Sr39, Sr47, SrAes $1 t$ and SrAes $7 t$ (summarized in Mago et al. 2013), and leaf rust (caused by P. triticina), namely Lr28, Lr35, Lr36, Lr47, Lr51 and Lr66 (summarized in McIntosh et al. 2013). To date, none of these genes are deployed in agriculture. This work describes the discovery of potentially new stem rust resistance genes in four different sources of $A e$. speltoides.

\section{Materials and Methods}

Diploid accessions of Aegilops speltoides AEG357-4 and AEG874-60 were provided courtesy of the The Harold and Adele Lieberman Germplasm Bank, Tel Aviv University, Israel. Amphidiploids of Chinese Spring/Ae. speltoides TS01 and TA8026 were provided courtesy of the Weizmann Institute, Rehovot, Israel and Wheat Genetics Resource Center, Kansas State University, USA, respectively.

Diploid Ae. speltoides accessions were crossed with cultivar Angas (from Dr Hugh Wallwork, Urrbrae, Australia) using wheat as the female parent. $F_{1}$ seedlings were treated with $0.07 \%$ colchicine (described in Dundas et al. 2008). Hybrids were crossed as females with Angas and later with $\mathrm{cv}$. Westonia up to $\mathrm{BC}_{5}$. The amphiploid accessions were crossed with Angas then backcrossed with Westonia as the female parent to $\mathrm{BC}_{5}$.

Identification of backcrossed plants carrying each of the Ae. speltoides S-genome chromosomes using RFLP markers has been described in Dundas et al. (2008). PCR markers for 2S chromosomes were obtained from Mago et al. (2009) (Sr39\#22r) and Seyfarth et al. (1999) (35R2/ BCD260F1). Backcrossed lines carrying S-genome chromosomes were tested for stem rust resistance at the University of Sydney (Pgt 34-1,2,3,4,5,6,7) and at Urrbrae (Pgt 343-1,2,3,5,6). Selected lines were screened against Ug99 races TTKSK, TTKST $(+S r 24)$ and TTTSK $(+S r 36)$ at the USDA Cereal Disease Laboratory, St. Paul, USA. Genomic in situ hybridization (GISH) was conducted on mitotic chromosome spreads using the procedure described in Mago et al. (2013). 


\section{Results and Discussion}

Ae. speltoides AEG357-4 Lines carrying the T2BS-2S\#3S.2S\#3L-2BL recombinant chromosome derived from AEG357-4 (Dundas et al. 2008) showed the loss of distal Ae. speltoides $2 \mathrm{~S} \# 3$ chromosome segments and the retention of proximal 2S\#3 chromosome segments (Figs. 5.1a and 5.2). This line was found to be resistant to stem rust pathotypes Pgt 34-1,2,3,4,5,6,7, Pgt 343-1,2,3,5,6 (Dundas et al. 2008) and TTKST (pers. comm., Professor Z. Pretorius, University of Free State, Bloemfontein, South Africa). After crossing this recombinant line with Sears' phlb mutant, we selected five lines derived from a $F_{3}$ population of 155 seedlings showing confirmed dissociation of RFLP and PCR markers specific for the 2S\#3 chromosome (Fig. 5.2). All lines showed resistance to Australian stem rust pathotypes and Ug99 races TTKSK, TTKST and TTTSK (Table 5.1). The dissociation plants \#3 and \#20 showed only 2S\#3S markers indicating that a stem rust resistance gene locates on the short arm of the 2S\#3 chromosome (Fig. 5.2). Dissociation plant \#27
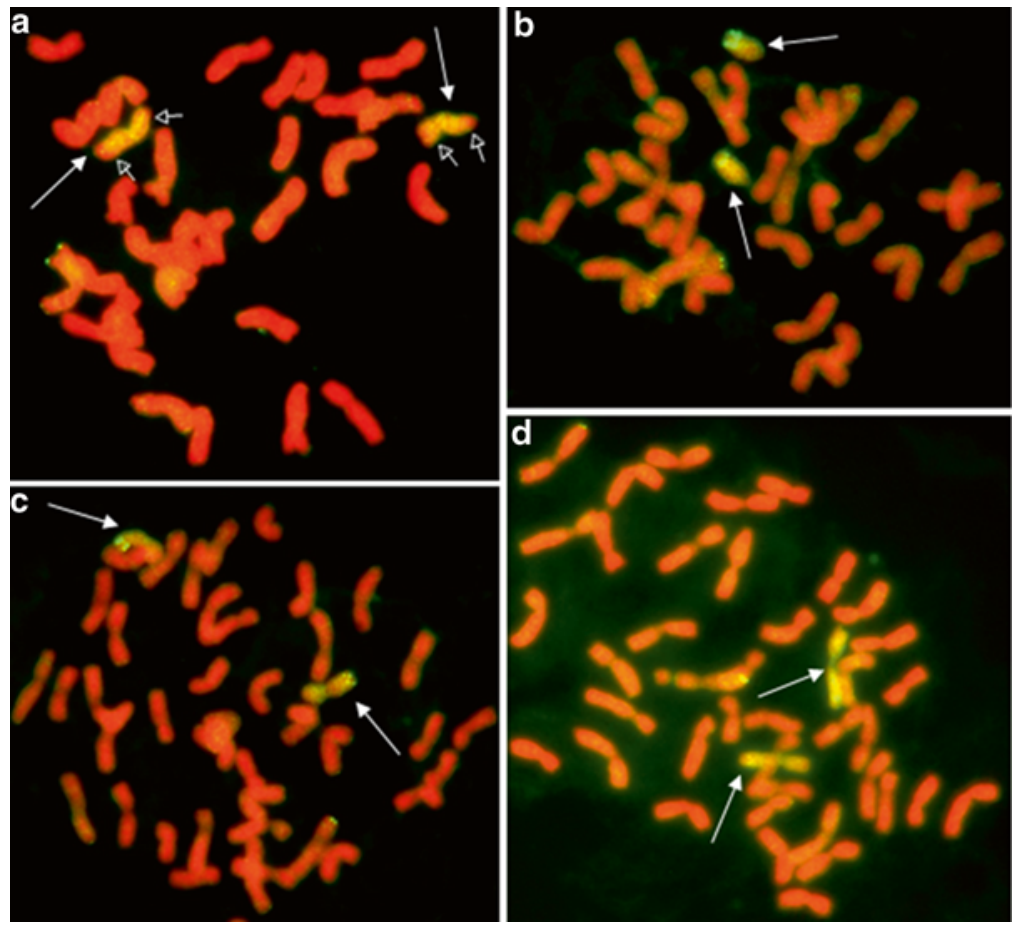

Fig. 5.1 Genomic in situ hybridization using Aegilops speltoides genomic DNA as probe on (a) T2BS-2S\#3S.2S\#3L-2BL translocation line, (b) 2S\#4L ditelocentric addition line, (c) 2S\#5 disomic addition line and (d) $2 \mathrm{~S} \# 6$ disomic addition line. Solid arrows show Ae. speltoides $2 \mathrm{~S}$ chromosome segments. Open arrows show translocation breakpoints 


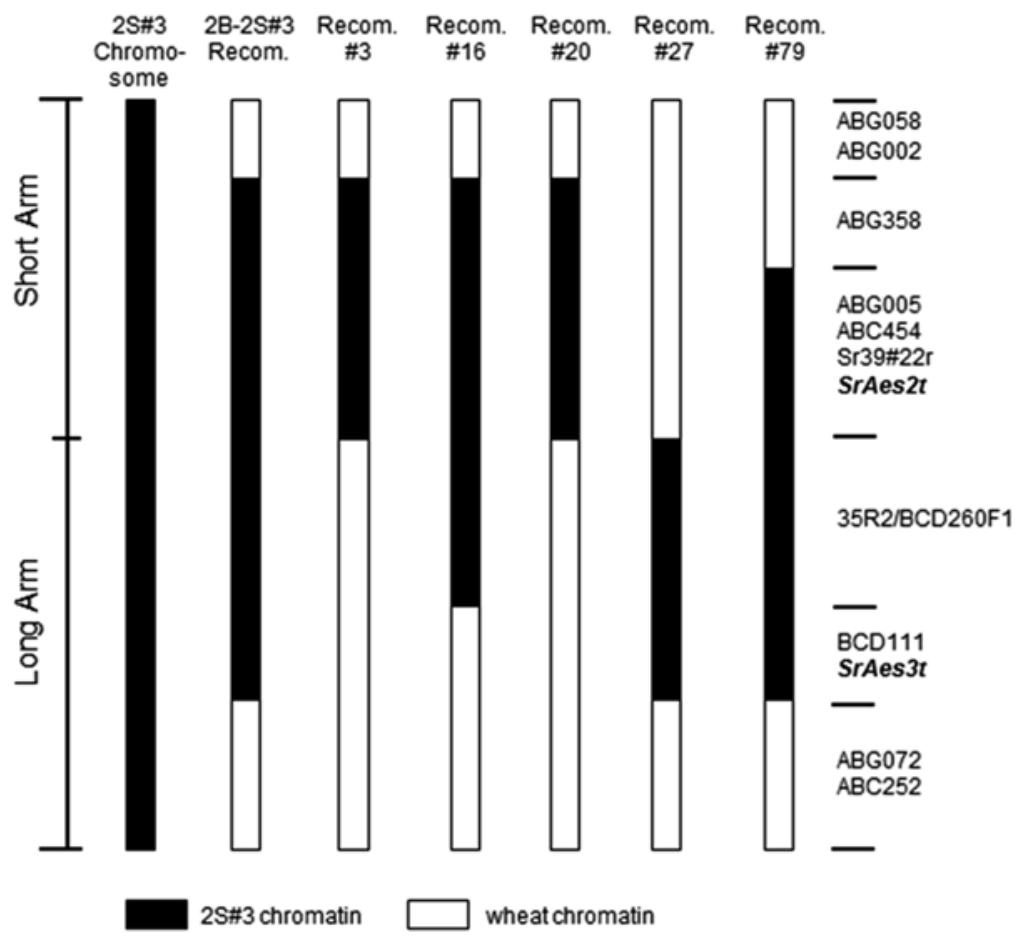

Fig. 5.2 Diagrammatic representation of wheat-chromosome $2 \mathrm{~S} \# 3$ recombinant lines showing the positions of RFLP and PCR markers and stem rust resistance genes. Aegilops speltoides and wheat chromatin are represented in black and white, respectively. The primary recombinant $2 \mathrm{~B}-2 \mathrm{~S} \# 3$ and secondary recombinants \#3, \#16, \#20, \#27 and \#79 are resistant to stem rust pathotype tested

Table 5.1 Stem rust infection types (ITs) of wheat lines carrying Aegilops speltoides $2 \mathrm{~S}$ chromosomes to Ug99 pathotypes TTKSK, TTKST and TTTSK, and two Australian pathotypes

\begin{tabular}{l|l|l|l|l|l}
\hline & \multicolumn{5}{l}{ Stem rust pathotype } \\
\cline { 2 - 7 } Line & $\begin{array}{l}\text { TTKSK } \\
\text { (Ug99) }\end{array}$ & $\begin{array}{l}\text { TTKST } \\
\text { (Ug99+Sr24) }\end{array}$ & $\begin{array}{l}\text { TTTSK } \\
\text { (Ug99+Sr36) }\end{array}$ & $\begin{array}{l}343-1,2, \\
3,5,6\end{array}$ & $\begin{array}{l}34-1,2,3,4, \\
5,6,7\end{array}$ \\
\hline Westonia+T2B-2S\#3 & & & $;$ & $2-$ \\
\hline Westonia+2S\#3 recomb \#3 & $2+$ & $22+$ & 2 & $;$ & \\
\hline Westonia+2S\#3 recomb \#16 & $; 2--$ & $; 2-$ & $; 2-$ & $;$ & \\
\hline Westonia+2S\#3 recomb \#20 & $; 2-$ & - & - & $;$ & \\
\hline Westonia+2S\#3 recomb \#27 & 2 & $2-$ & $2-$ & $;$ & \\
\hline Westonia+2S\#3 recomb \#79 & 2 & $; 2-$ & $; 2-$ & $;$ & \\
\hline Westonia+2S\#4 addition & $; / ; N$ & $; 1$ & $; 1$ & $;$ & \\
\hline Westonia+2S\#5 addition & $; 1$ & $; / 2-$ & $; / 2-$ & $;$ & \\
\hline Westonia+2S\#6 addition & & & & $;$ & $2-$ \\
\hline Westonia & 4 & 4 & 4 & 4 & \\
\hline Angas & 3 & $3+$ & $3+$ & & \\
\hline
\end{tabular}

The 2S\#3, 2S\#4, 2S\#5 and 2S\#6 chromosomes were derived from diploid line AEG357-4, CS/Ae. speltoides amphiploid TA8026, CS/Ae. speltoides amphiploid TS01 and diploid line AEG874-60, respectively. An IT of ' 3 ' or ' 4 ' is susceptible 
showed only $2 \mathrm{~S} \# 3 \mathrm{~L}$ markers suggesting that a stem rust resistance gene is located on the long arm of the $2 \mathrm{~S} \# 3$ chromosome (Fig. 5.2).

GISH studies on the five stem rust resistant secondary recombinants failed to reveal $2 \mathrm{~S} \# 3$ chromatin, which suggested that very small segments of $2 \mathrm{~S} \# 3$ chromatin were present on these secondary recombinants. We temporarily name the resistance gene on the short arm of the $2 \mathrm{S \#} 3$ chromosome as $\operatorname{SrAes} 2 t$ and the gene on the long arm as $\operatorname{SrAes} 3 t$.

Chinese Spring/Ae. speltoides Amphiploid TA8026 Stem rust resistant backcrossed lines derived from TA8026 were isolated which carried only the group 2 long arm RFLP markers BCD111, ABG072 and ABC252 specific for the 2S\#4 chromosome. Group 2 short arm probes ABG058, BCD221 and ABC454 did not detect the presence of $2 \mathrm{~S}$ chromatin. GISH analysis of these lines showed the presence of either a wheat-2S\#4L translocation or $2 \mathrm{~S} \# 4 \mathrm{~L}$ telocentric chromosome (Fig. 5.1b). The line carrying the telocentric 2S\#4L chromosome was resistant to Australian and Ug99 stem rust races (Table 5.1). The stem rust resistance gene on the $2 \mathrm{~S} \# 4 \mathrm{~L}$ chromosome has been temporarily named SrAes $4 t$.

Chinese Spring/Ae. speltoides Amphiploid TS01 Backcrossed lines derived from the amphiploid TS01 were isolated which carried group 2 RFLP markers for the probes ABG002, ABC358 and ABC454, the PCR marker Sr39\#22r (short arm), and RFLP markers for probes BCD111, ABG072 and PCR marker 35R2/ BCD260F1 (long arm), specific for the 2S\#5 chromosome. Rust tests showed these lines to be resistant to Australian stem rust and Ug99 pathotypes (Table 5.1). GISH analysis of the line showed the presence of an apparently entire Ae. speltoides $2 \mathrm{S \# 5}$ chromosome (Fig. 5.1c). The stem rust resistance gene on that chromosome is here named as $\operatorname{SrAes} 5 t$.

Ae. speltoides AEG874-60 Dundas et al. (2008) reported that the diploid accession AEG874-60 was resistant to stem rust races Pgt 34-2,12,13, Pgt 34-1,2,3,4,5,6,7 and $P g t$ 98-1,2,3,5,6. BC B $_{5}$ plants with cv. Westonia were isolated carrying only the 2S\#6 chromosome from AEG874-60 and carried Ae. speltoides-specific markers ABG058, BCD221, ABG002, ABG358, ABC454 and Sr39\#22r (short arm) and BCD111, ABG072, ABC252 and 35R2/BCD260F1 (long arm). Plants with the 2S\#6 chromosome were resistant to stem rust Pgt 34-1,2,3,4,5,6,7 and Pgt 3431,2,3,5,6 (Table 5.1). GISH analysis showed an entire 2S\#6 chromosome present (Fig. 5.1d). The stem rust resistance gene on that chromosome is here named as SrAes6t.

It is unknown if the stem rust resistance genes described herein are different from those previously reported on Ae. speltoides $2 \mathrm{~S}$ chromosomes (namely Sr32, SrAes 1 , SrAes $7 t, S r 39$ and Sr47). Lines carrying the T2BS-2S\#3S.2S\#3L-2BL chromosome with genes $\operatorname{SrAes} 2 t$ and $S r A e s 3 t$ are being distributed to many wheat breeding programs around the World. 
Acknowledgments This work was partly funded by the Grains Research and Development Corporation (Australia), and the Bill and Melinda Gates Foundation (USA) and DFID (UK) through a sub-agreement with Cornell University (USA). We thank Dr. Moshe Feldman (Weizmann Institute, Rehovot, Israel) for providing seeds of amphiploid TS01.

Open Access This chapter is distributed under the terms of the Creative Commons Attribution Noncommercial License, which permits any noncommercial use, distribution, and reproduction in any medium, provided the original author(s) and source are credited.

\section{References}

Dundas IS, Verlin DC, Park RF et al (2008) Rust resistance in Aegilops speltoides var. ligustica. In: Appels $\mathrm{R}$ et al (eds) Proceedings of the 11th international wheat genetics symposium, Brisbane, Australia, vol 1, pp 200-202

Kislev ME (1982) Stem rust of wheat 3300 years old found in Israel. Science 216:993-994

Mago R, Zhang P, Bariana HS et al (2009) Development of wheat lines carrying stem rust resistance gene Sr39 with reduced Aegilops speltoides chromatin and a simple PCR marker for marker-assisted selection of the gene. Theor Appl Genet 119:1441-1450

Mago R, Verlin D, Zhang P et al (2013) Development of wheat-Aegilops speltoides recombinants and simple PCR-based markers for stem rust resistance genes on the $2 \mathrm{S \# 1}$ chromosome. Theor Appl Genet 126:2943-2955

McIntosh RA, Yamazaki Y, Dubcovsky J et al (2013) Catalogue of gene symbols for wheat. In: Proceedings of the 12th international wheat genetics symposium, Yokohama

Seyfarth R, Feuillet C, Schachermayr G et al (1999) Development of a molecular marker for the adult plant leaf rust resistance gene Lr35 in wheat. Theor Appl Genet 99:554-560 